\title{
Synthesis characterization and in vitro drug release from acrylamide and sodium alginate based superporous hydrogel devices
}

\author{
Manju Nagpal, Shailendra Kumar Singh ${ }^{1}$, Dinanath Mishra ${ }^{1}$ \\ Department of Pharmaceutics, Chitkara College of Pharmacy, Chitkara University, Patiala, Punjab, 'Department of Pharmaceutical Sciences, \\ Guru Jambheshwar University of Science and Technology, Hisar, Haryana, India
}

\begin{abstract}
Objective: Present investigation was aimed at developing gastroretentive superporous hydrogels (SPHs) having desired mechanical characteristics with sustained release. Materials and Methods: The acrylamide based SPHs of various generations $\left(1^{\text {st }}, 2^{\text {nd }}\right.$ and $\left.3^{\text {rd }}\right)$ were synthesized by gas blowing technique. The prepared SPHs were evaluated for swelling, mechanical strength studies and scanning electron microscopy studies. Verapamil hydrochloride was loaded into selected SPHs by aqueous drug loading method and characterized via Fourier transform infrared spectroscopy (FTIR), X-ray diffraction (X-RD), differential scanning calorimetry (DSC), nuclear magnetic resonance (NMR) and in vitro drug release studies. Results: SPHs of third generation were observed to have desired mechanical strength with sufficient swelling properties. Integrity of the drug was maintained in hydrogel polymeric network as indicated by FTIR, X-RD, and DSC and NMR studies. Initially, fast drug release (up to $60 \%$ ) was observed in 30 min in formulation batches containing pure drug only ( $A, C$ and $E$ ), which was further sustained untill $24 \mathrm{~h}$. Discussion: The increase in mechanical strength was due to the chemical cross-linking of secondary polymer in hydrogel network. The initial burst release was due to the presence of free drug at the surface and later sustained drug release was due to diffusion of entrapped drug in polymeric network. Significant decrease in drug release was observed by the addition of hydroxypropyl methyl cellulose. Conclusion: SPH interpenetrating networks with fast swelling and sufficient mechanical strength were prepared, which can be potentially exploited for designing gastroretentive drug delivery devices.
\end{abstract}

Key words: Capillary channels, gastroretentive drug delivery, interpenetrating networks, mechanical strength, swelling properties

\section{INTRODUCTION}

Drug delivery technologies are as important as new chemical entities entering into the pharmaceutical industries allowing more effective use of existing drugs and successful development of new drug candidates. Hydrogels have long been established in drug delivery to control the release of drug from a conventional solid dosage form. ${ }^{[1]}$ Hydrogels are polymeric materials with open

\section{Address for correspondence:}

Prof. Dinanath Mishra,

Department of Pharmaceutical Sciences, Guru Jambheshwar

University of Science and Technology, Hisar - 125001 ,

Haryana, India.

E-mail: drdnmishra@yahoo.com

\begin{tabular}{|l|l|}
\hline \multicolumn{2}{|c|}{ Access this article online } \\
\hline Quick Response Code: & Website: \\
\hline & www.jpionline.org \\
\cline { 2 - 2 } & DOI: \\
\hline
\end{tabular}

structures/pores with the ability to imbibe large quantities of water and solutes. Biocompatible polymer hydrogels have found wide application in biomedicine, agriculture and food processing industry. ${ }^{[2]}$ The slow swelling of these non-porous rigid hydrogels and low loading capacities restrict their use in pharmaceutical applications where fast swelling is required. A new generation of hydrogels, i.e., superporous hydrogels (SPHs) are a 3D network of hydrophilic polymers that absorb a considerable amount of water in a very short period of time due to the presence of many pores with a diameter of micron to millimeter range. ${ }^{[3]}$ Gas blowing technique employing foaming agents (sodium bicarbonate) is used to synthesize these porous SPHs. ${ }^{[4,5]}$ Several important properties of SPHs such as fast swelling, large swelling ratio and surface slipperiness make them excellent candidate to develop gastric retention devices. Furthermore, main limitation with SPHs for gastroretentive purpose is poor mechanical strength. The mechanical properties can be significantly enhanced by adding cross-linked polymers thereby creating interpenetrating networks. Interpenetrating polymeric networks comprise of two or more independent polymeric network that is formed in the presence of one another. ${ }^{[6]}$ Chitosan, chitin, gelatin and alginates are widely used natural polymers while polyacrylamide, 
poly (acrylic acid), poly (vinylpyrrolidone) is among synthetic one used to generate superporous hydrogel interpenetrating networks (SPHIPNs). ${ }^{[7-10]}$ Alginates are linear, non-branched polysaccharides containing varying amounts of 1, 4'-linked $\beta$-D-mannuronic acid and $\alpha$-L-guluronic acid residues. The alginate chains can dimerize to form gel networks in the presence of divalent or trivalent metal cations. Water soluble alginate polymer was added to monomer mixture before gelation and foaming to yield polymerized SPH, which was treated with metal cations to induce metal complexation of alginate portions of SPH structure. ${ }^{[1]}$ Verapamil hydrochloride is a calcium channel blocker; (biopharmaceutic classification scheme) class II drug. Verapamil shows high pharmacokinetic variability mainly due to its extensive first pass effect. With an elimination half-life of 1-2 h, it is a potential candidate for controlled release drug delivery. Controlled or sustained release of verapamil from novel hydrogel drug delivery systems improves patient compliance by reducing the dosing frequency because it is used for chronic disease. Moreover, the side effects and therapeutic effects are also improved. ${ }^{[12,13]}$

This paper describes the synthesis and characterization of various generations of SPHs using the polyacrylamide (primary polymer) and sodium alginate (secondary polymer) incorporating verapamil hydrochloride as a model drug.

\section{MATERIALS AND METHODS}

\section{Materials}

Verapamil hydrochloride was kindly gifted by Abott Healthcare Pvt. Ltd. Baddi, India; Acrylic acid and acrylamide was purchased from CDH Pvt. Ltd., India; Pluronic F 127 (PF 127) was purchased from sigma life sciences; N,Nmethylenebisacrylamide (BIS), ammonium per sulfate (APS), N,N,N',N'-tetraethylmethylenediamine (TEMED), sodium alginate and sodium bicarbonate were purchased from Loba Chemie, Mumbai, India. All chemicals used were of suitable analytical grade and used as received.

\section{Methods \\ Preparation of SPHs by gas blowing technique}

Gas blowing technique ${ }^{[4]}$ with minor modification was used to synthesize SPH based on polyacrylamide. Weighed quantities of all ingredients including monomers (acrylamide), cross-linker (BIS), foam stabilizer (PF 127), acrylic acid, reaction initiator pair (APS-TEMED) were taken in the test tube $(15 \mathrm{~mm}$ outer diameter) [Table 1] and test tube was shaken thoroughly using a vortex mixer after addition of each ingredient. $\mathrm{pH}$ of monomer solution was adjusted to $5.5 \pm 0.5$ using the acrylic acid followed by the addition of sodium bicarbonate with continuous stirring. Immediate stirring is required to uniformly distribute generating gas bubbles. Thorough mixing at this step leads to simultaneous foaming and gelation thereby yielding conventional superporous hydrogel (CSPH) i.e. first generation SPH.

$\begin{aligned} & \text { Table 1: Composition of various generations } \\
& \text { of superporous hydrogels }\end{aligned}$
\begin{tabular}{lccc}
\hline Name of ingredient & CSPH & SPHC & SPHIPN \\
\hline Acrylamide (50\%) & $500 \mu \mathrm{l}$ & $500 \mu \mathrm{l}$ & $500 \mu \mathrm{l}$ \\
N,N-methylenebisacrylamide & $200 \mu \mathrm{l}$ & $200 \mu \mathrm{l}$ & $200 \mu \mathrm{l}$ \\
Pluronic F 127 (10\%) & $100 \mu \mathrm{l}$ & $100 \mu \mathrm{l}$ & $100 \mu \mathrm{l}$ \\
Distilled water & $460 \mu \mathrm{l}$ & $460 \mu \mathrm{l}$ & - \\
Acrylic acid (pH-5-6) & $25 \mu \mathrm{l}$ & $25 \mu \mathrm{l}$ & $25 \mu \mathrm{l}$ \\
Na alginate (2\%) & - & $500 \mu \mathrm{l}$ & $500 \mu \mathrm{l}$ \\
Ammonium per sulfate (20\%) & $50 \mu \mathrm{l}$ & $50 \mu \mathrm{l}$ & $50 \mu \mathrm{l}$ \\
N,N,N',N'-tetraethylmethylenediamine & $50 \mu \mathrm{l}$ & $50 \mu \mathrm{l}$ & $50 \mu \mathrm{l}$ \\
$(20 \%)$ & & & \\
NaHCO & $100 \mathrm{mg}$ & $100 \mathrm{mg}$ & $100 \mathrm{mg}$ \\
Treatment with CaCl & - & - & $5-10 \%$ \\
& & & for $1.5 \mathrm{~h}$ \\
\hline
\end{tabular}

CSPH: Conventional superporous hydrogel, SPHC: Superporous hydrogel composites, SPHIPN: Superporous hydrogel interpenetrating network

For preparation of superporous hydrogel composites (SPHC) i.e. second generation SPH, Na Alginate (secondary polymer) was added prior to addition of APS-TEMED (reaction initiator pair).

For preparation of SPHIPNs i.e. third generation SPH, SPHC was further treated with calcium chloride solution for $1.5 \mathrm{~h}$.

\section{Effect of drying conditions}

To evaluate the effect of drying on porous network of SPH, the $\mathrm{SPH}$ were given different drying conditions - (i) oven drying at $50-55^{\circ} \mathrm{C}$ overnight; (ii) ethanol dehydration for $2 \mathrm{~h}$ followed by oven drying at $50-55^{\circ} \mathrm{C}$; (iii) freeze drying.

\section{Evaluation of SPHs}

\section{In situ evaluation of SPHs during synthesis}

All SPH samples were observed for texture, color, transparency, brittleness, flexibility, stickiness etc.

\section{Evaluation after synthesis}

\section{Scanning electron microscopy (SEM)}

The dried SPH were cut in transverse section and mounted on a double sided tape on aluminum stubs and were sputter coated with gold using fine coat ion sputter (JEOL) and then micrographs were recorded using scanning electron microscope (JEOL, JSM-6100) to study the effect of various drying conditions on porosity of hydrogels.

\section{Equilibrium swelling ratio}

Completely dried SPH was weighed and kept in excess of swelling medium (distilled water at $37^{\circ} \mathrm{C}$ ) until the equilibrium swelling was achieved and the hydrogel sample was again weighed. ${ }^{[14]}$ Ethanol dehydrated samples were selected for evaluation of different generation of SPHs. The experiments were carried out in triplicate and results are shown as mean $\pm \mathrm{SD}$.

The equilibrium swelling ratio was calculated as:

$\mathrm{Q}=\left(\mathrm{M}_{\mathrm{s}}-\mathrm{M}_{\mathrm{d}}\right) / \mathrm{M}_{\mathrm{d}}$ 
Where, $\mathrm{Q}$ is the equilibrium swelling ratio, $\mathrm{M}_{\mathrm{s}}$ is the mass of $\mathrm{SPH}$ in swollen state (till equilibrium swelling), $\mathrm{M}_{\mathrm{d}}$ is the mass of the SPH in the dried state.

\section{Equilibrium swelling time}

The SPH samples were kept in excess of swelling medium (distilled water at $37^{\circ} \mathrm{C}$ ) and the time required for equilibrium swelling was noted. ${ }^{[14]}$ It was also determined in triplicate for all batches.

\section{Density}

Solvent displacement method was used for the determination of density. The pre-weighed SPH sample was immersed in predetermined volume of hexane in a graduated cylinder. The increase in hexane volume was noted as volume of the solvent displaced or the volume of SPH sample. ${ }^{[15]}$ The experiments were carried out in triplicate and the results are shown as average \pm SD. Density was calculated using the formula:

Density $=$ Mass of superporous hydrogel/Volume of solvent displaced.

\section{Porosity}

Dried SPH were immersed in hexane overnight and weighed after excess amount of hexane on the surface was blotted. ${ }^{[15]}$ The experiments were carried out in triplicate and the results are shown as average \pm SD. Porosity was calculated using the formula:

Porosity $=\mathrm{V}_{\mathrm{p}} / \mathrm{V}_{\mathrm{t}}$

Where, $\mathrm{V}_{\mathrm{t}}$ is the total volume of $\mathrm{SPH}, \mathrm{V}_{\mathrm{p}}$ is the pore volume of $\mathrm{SPH}\left(\mathrm{V}_{\mathrm{t}}-\mathrm{V}_{\mathrm{l}}\right), \mathrm{V}_{1}$ is the volume of liquid displaced.

\section{Void fraction}

It was determined by immersing the $\mathrm{SPH}$ in $\mathrm{HCl}(\mathrm{pH} 1.2)$ up to equilibrium swelling. ${ }^{[16]}$ The dimensions of the swollen SPH were measured and by using these values, SPH sample volume was determined as the dimensional volume. The amount of absorbed $\mathrm{HCl}$ into $\mathrm{SPH}$ was determined by subtracting the weight of dried SPH sample from the weight of swollen SPH and the resulting values were assigned as the total volume of pores in the hydrogels. Void fraction was calculated using the formula:

Void Fraction = Dimensional Volume of superporous hydrogel/ Total volume of pores

All results were obtained in triplicate.

\section{Mechanical properties}

Compression force $(\mathrm{N})$ was determined using the TA-TX plus texture analyzer (stable micro systems) using a cylindrical aluminum probe (P75) having a pretest speed of $2.00 \mathrm{~mm} / \mathrm{s}$, test speed of $1 \mathrm{~mm} / \mathrm{s}$ and posttest speed of $2 \mathrm{~mm} / \mathrm{s}$ up to a distance of $3 \mathrm{~mm}$. The swollen SPH sample was placed on a disk shaped platform. Compression force was estimated as the peak value in the force versus time plot.

\begin{tabular}{|c|c|c|c|}
\hline Formulation batch & Drug (mg) & HPMC (mg) & $\mathrm{CaCl}_{2} \%$ \\
\hline$A$ & 120 & - & 5 \\
\hline $\mathrm{B}$ & 120 & 120 & 5 \\
\hline C & 120 & - & 7.5 \\
\hline $\mathrm{D}$ & 120 & 120 & 7.5 \\
\hline$E$ & 120 & - & 10 \\
\hline $\mathrm{F}$ & 120 & 120 & 10 \\
\hline
\end{tabular}

HPMC: Hydroxypropyl methyl cellulose

\section{Drug loading into SPHIPNs}

Ethanol dehydrated SPHIPNs were used for drug loading (Verapamil $\mathrm{HCl}$ ) owing to their mechanical strength and swelling behavior. The method of soaking or equilibration was used for drug loading. ${ }^{[17]}$ The amount of water required for complete swelling was determined and thereafter drug was dissolved in the predetermined amount of water. The SPH was kept in the drug solution and left until all solution was sucked up. Finally, the completely swollen hydrogel was freeze dried for $24 \mathrm{~h}$. Six drug loaded batches were prepared to evaluate the effect of cross-linker concentration $(5 \%, 7.5 \%$ and $10 \%)$ on the drug release (Batch $\mathrm{A}, \mathrm{C}$ and $\mathrm{E}$ respectively) and to evaluate the effect of addition of other polymer (on drug release) in above formulations by taking drug: Hydroxy propyl methyl cellulose (HPMC) ratio of 1:1 in batches B, D and F [Table 2].Evaluation of drug loaded SPHIPNs

\section{Drug loading capacity}

Drug loaded SPHIPN sample was triturated and weight equivalent to $10 \mathrm{mg}$ of the drug was dissolved in $100 \mathrm{ml}$ of simulated gastric fluid (SGF) $(\mathrm{pH} 1.2)$ and the solution was filtered and then analyzed spectrophotometrically at $278 \mathrm{~nm}$.

Drug loading capacity: $\mathrm{A} / \mathrm{b}^{*} 100$

where, a is observed amount of the drug; b is the theoretical amount of the drug present in hydrogel.

\section{Differential scanning calorimetry (DSC)}

DSC studies were carried out to study the physical integrity of the drug with in the polymeric network. The DSC thermogram of the pure drug and drug loaded hydrogel was obtained by using DSC instrument (DSC Q10 V 9.9 Build 303). Samples were heated under nitrogen atmosphere on an aluminum pan at a rate of $10^{\circ} \mathrm{C} / \mathrm{min}$. over the temperature range of $50-400^{\circ} \mathrm{C}$.

\section{Fourier transform infrared spectroscopy (FTIR)}

Dried drug loaded SPHIPN were powdered by using a mortar and pestle. The IR spectra were recorded by using $\mathrm{KBr}$ pellet method over the scanning range of $4000-400 \mathrm{~cm}^{1}$ using FTIR spectrophotometer (Perkin Elmer Spectrum 400). The FTIR spectrum was recorded to study the interaction of drug with the components of polymeric network. 


\section{X-ray diffraction (X-RD)}

The $\mathrm{X}-\mathrm{RD}$ analysis was carried out to monitor the changes in crystallinity characteristics of the drug when the drug was loaded into SPH interpenetrating networks. The drug loaded SPHIPN was powdered in a mortar and then the X-RD patterns were measured using X-ray diffractometer (X'pert PRO, PAN analytical, Netherland) using Ni filtered, $\mathrm{CuK} \alpha$ radiation with a voltage of $45 \mathrm{kV}$ and $40 \mathrm{~mA}$ current.

\section{${ }^{1} \mathrm{H}$ nuclear magnetic resonance (NMR) studies}

${ }^{1} \mathrm{H}$ NMR studies were carried out using a cryo-magnet spectrometer $400 \mathrm{MHz}$ Bruker Fourier transform-NMR spectrometer (Bruker) using $\mathrm{D}_{2} \mathrm{O}$ and chemical shifts were recorded in ppm downfield from internal reference tetramethylsilane.

\section{In Vitro drug release}

The in vitro drug release of verapamil hydrochloride from various batches of SPHIPNs was determined using United States Pharmacopeia Dissolution Type II apparatus (Paddle Type) at $37 \pm 0.5^{\circ} \mathrm{C}$ at a paddle speed of $50 \mathrm{rpm}$ in $900 \mathrm{ml}$ of SGF ( $\mathrm{pH}$ 1.2) for $24 \mathrm{~h}^{\left[{ }^{[17]}\right.}$ At specified intervals, $10 \mathrm{ml}$ of the dissolution medium was withdrawn and an equivalent volume of fresh dissolution medium was replaced. The samples were analyzed at $278 \mathrm{~nm}$ using the ultra violet-visible spectrophotometer (Shimadzu, Japan).

\section{RESULTS}

In situ evaluation during synthesis

Various generations of SPHs were observed for physical changes during the various steps of synthesis. The results are depicted in Table 3 and shown in Figure 1. Evaluation after synthesis

\section{SEM}

The SEM photographs of ethanol dehydrated, freeze dried and air dried SPHs samples are shown in Figure 2. The

\begin{tabular}{|c|c|c|c|}
\hline $\begin{array}{l}\text { Type of } \\
\text { SPH } \rightarrow \text { physical } \\
\text { parameter } \downarrow\end{array}$ & $\mathrm{CSPH}$ & SPHC & SPHIPNs \\
\hline \multicolumn{4}{|l|}{ During synthesis } \\
\hline Texture & $\begin{array}{l}\text { Soft, sticky, } \\
\text { less flexible }\end{array}$ & $\begin{array}{l}\text { Soft, less } \\
\text { flexible }\end{array}$ & Soft, flexible \\
\hline Color & $\begin{array}{l}\text { Completely } \\
\text { white }\end{array}$ & $\begin{array}{l}\text { Creamish } \\
\text { white }\end{array}$ & $\begin{array}{l}\text { Creamish } \\
\text { white }\end{array}$ \\
\hline $\begin{array}{l}\text { During ethanol } \\
\text { dehydration/ } \\
\text { cross-linker } \\
\text { treatment }\end{array}$ & $\begin{array}{l}\text { No immediate } \\
\text { hardening }\end{array}$ & $\begin{array}{l}\text { Hard and } \\
\text { brittle }\end{array}$ & Hard \\
\hline After drying & Hard and sticky & Non-sticky & Non-sticky \\
\hline After swelling & $\begin{array}{l}\text { Completely } \\
\text { transparent }\end{array}$ & $\begin{array}{l}\text { No complete } \\
\text { transparency }\end{array}$ & $\begin{array}{l}\text { Creamish } \\
\text { opaque }\end{array}$ \\
\hline
\end{tabular}

SPHs: Superporous hydrogels, CSPH: Conventional superporous hydrogel, SPHC: Superporous hydrogel composites, SPHIPNs: Superporous hydrogel interpenetrating networks
SEM images revealed more uniform porous structure with interconnected channels in ethanol dehydrated SPHs. With freeze dried SPHs, capillary channels were observed, but were not as well-defined as in ethanol treated batches. However, distorted and irregular capillary channels were observed in air dried SPHs. Freeze drying resulted in fluffy (light weight) SPHs as compared to ethanol dehydrated and air dried batches.

\section{Equilibrium swelling ratio}

Equilibrium swelling ratio was found to be highest (130.57 \pm $0.667)$ with CSPH batches as compared to SPHC (112 \pm 0.145$)$. A significant decrease in swelling ratio of various SPHIPNs (5\%, $7.5 \%$ and $10 \%$ ) was observed as compared with that in $\mathrm{CSPH}$ and SPHC [Table 4].

\section{Equilibrium swelling time}

Equilibrium swelling time was observed to be less than 5 min in CSPH and SPHC. However, various SPHIPNs took more time (up to $30 \mathrm{~min}$ ) for equilibrium swelling, in comparison to CSPH and SPHC [Table 4].

\section{Density}

The density of all the SPH batches was observed to be less than 1 i.e., all of them were able to float in water [Table 4].
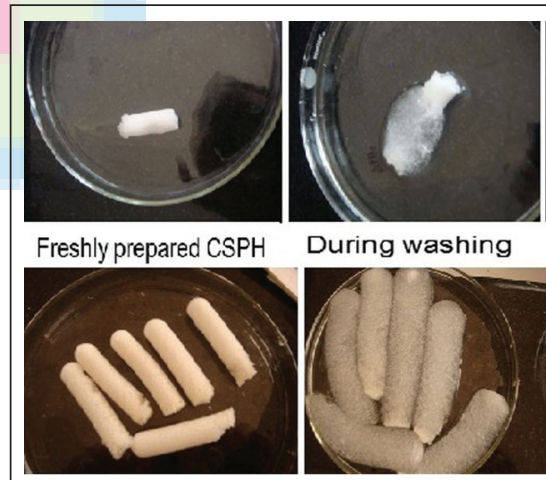

During washing
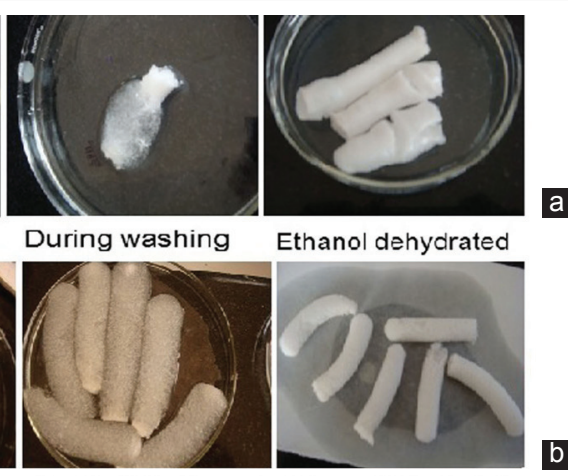

Freshly prepared SPHC During Washing Ethanol dehydrated

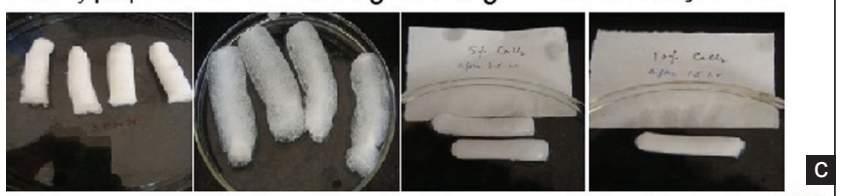

Freshly prepared SPHIPN During washing Crosslinker treatment-5\% Crosslinker treatment-10\%
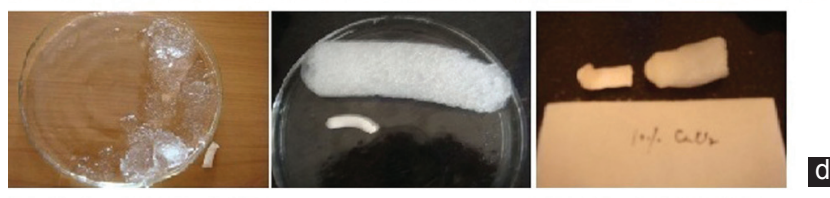

Swelled and dried CSPH Svielled and dried SPHC Swelled and Dried SPHIPA

Figure 1: Various steps during synthesis of different generation superporous hydrogel (a) conventional superporous hydrogel (CSPH), (b) superporous hydrogel composites (c) superporous hydrogel interpenetrating networks (SPHIPNs), (d) swelled and dried CSPH, SPHC and SPHIPN 


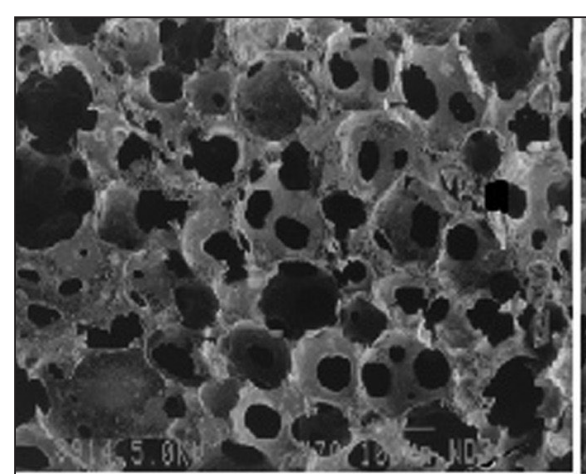

Ethanol Dehydrated

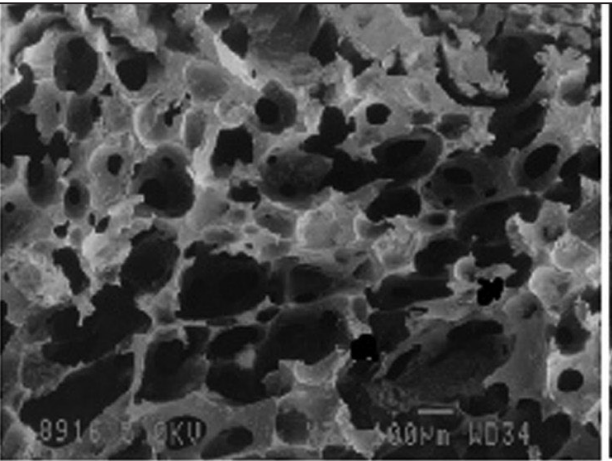

Freeze Dried

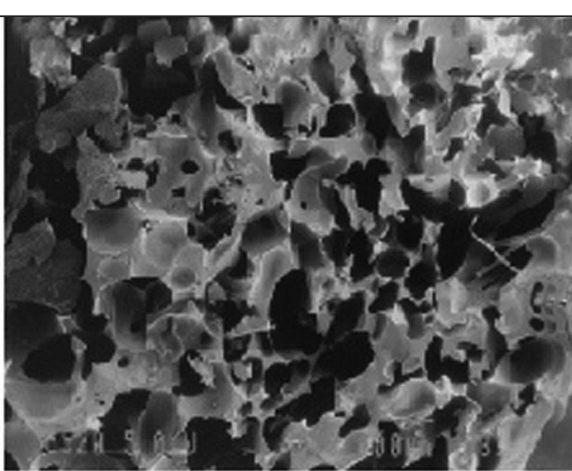

Oven Dried

Figure 2: Scanning electron micrographs of ethanol dehydrated, freeze dried and oven dried superporous hydrogels

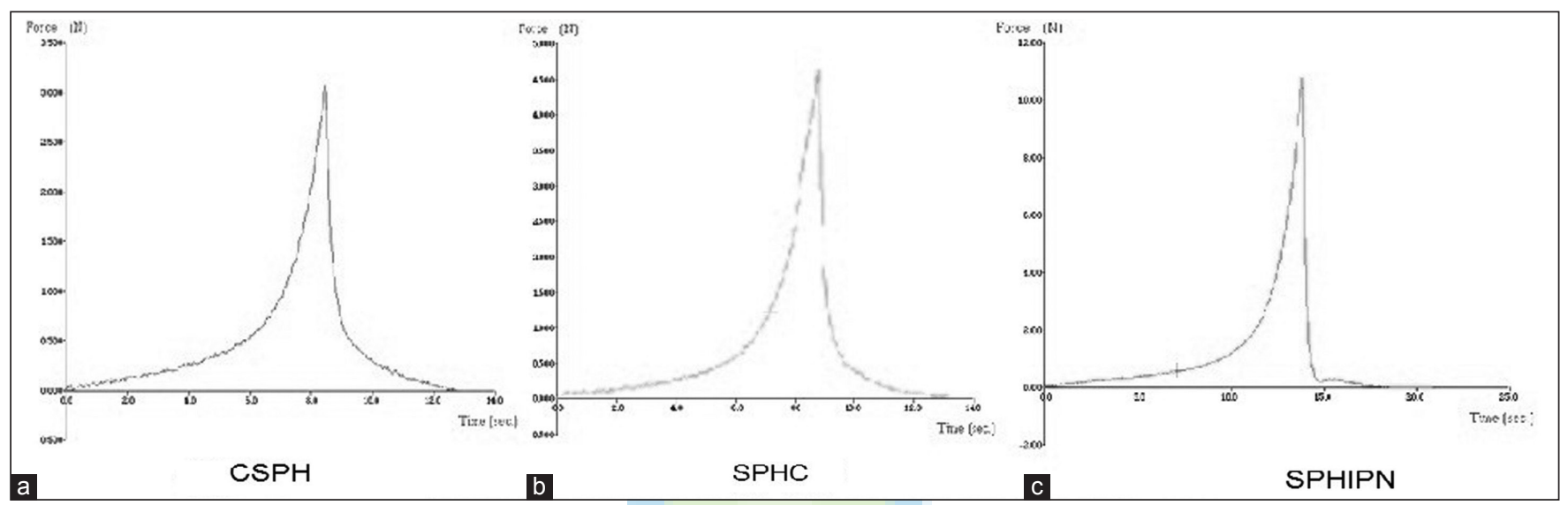

Figure 3: Mechanical strength of various generations of superporous hydrogels (a) conventional superporous hydrogel (b) superporous hydrogel composites (c) superporous hydrogel interpenetrating networks

\begin{tabular}{|c|c|c|c|c|c|}
\hline \multirow[t]{2}{*}{ Type of SPH $\rightarrow$ parameter $\downarrow$} & \multirow[t]{2}{*}{ CSPH } & \multirow[t]{2}{*}{ SPHC } & \multicolumn{3}{|c|}{ SPHIPNs } \\
\hline & & & $\begin{array}{c}\text { Treatment } \\
\text { with } 5 \% \mathrm{CaCl}_{2}\end{array}$ & $\begin{array}{c}\text { Treatment } \\
\text { with } 7.5 \% \mathrm{CaCl}_{2}\end{array}$ & $\begin{array}{c}\text { Treatment } \\
\text { with } 10 \% \mathrm{CaCl}_{2}\end{array}$ \\
\hline Equilibrium swelling ratio & $130.57 \pm 0.667$ & $112 \pm 0.145$ & $36.67 \pm 0.53$ & $27.43 \pm 0.67$ & $8.3 \pm 0.57$ \\
\hline Equilibrium swelling time (min) & $3-5$ & $2-3$ & $8-10$ & $20-25$ & $\geq 30 \mathrm{~min}$ \\
\hline Density & $0.87 \pm 0.014$ & $0.56 \pm 0.03$ & $0.66 \pm 0.024$ & $0.70 \pm 0.025$ & $0.71 \pm 0.012$ \\
\hline Porosity & $42.31 \pm 3.4$ & $45.6 \pm 1.75$ & $44.97 \pm 0.67$ & $32.69 \pm 0.78$ & $30 \pm 0.02$ \\
\hline Void fraction & $0.934 \pm 0.06$ & $1.79 \pm 0.154$ & $1.79 \pm 0.045$ & $0.95 \pm 0.12$ & $0.75 \pm 0.04$ \\
\hline
\end{tabular}

SPH: Superporous hydrogel, CSPH: Conventional superporous hydrogel, SPHC: Superporous hydrogel composites, SPHIPNs: Superporous hydrogel interpenetrating networks

\section{Porosity}

The porosity was observed to be $42.31 \pm 3.4 \%$ in case of SPHC batches, $45.6 \pm 1.75 \%$ in SPHC and $44.97 \pm 0.67 \%, 32.69 \pm$ $0.78 \%, 30 \pm 0.02$ in SPHIPNs batches $\left(5 \%, 7.5 \%\right.$ and $10 \% \mathrm{CaCl}_{2}$ respectively) [Table 4]

\section{Void fraction}

The results of void fraction [Table 4] was found to be $0.934 \pm$ $0.06,1.79 \pm 0.154,1.79 \pm 0.045,0.95 \pm 0.12$ and $0.75 \pm 0.04$ in CSPH, SPHC and SPHIPNs batches $(5 \%, 7.5 \%$ and $10 \%$ $\mathrm{CaCl}_{2}$ ) respectively.

\section{Mechanical strength}

Appropriate mechanical strength should be provided by the hydrogel for their application as the gastric retention device. Compression strength of CSPH was observed as equivalent to $3 \mathrm{~N}$ [Figure 3a] and increase in mechanical strength was observed by addition of second polymer [Figure $3 \mathrm{~b}$ and $\mathrm{c}$ ]. Cross-linking of second polymer leads to significant enhancement (equivalent to $10 \mathrm{~N}$ ) in mechanical strength of SPHs as observed in SPHIPNs.

\section{Drug loading capacity}

The drug loading capacity was observed to be $90 \pm 1.67-95 \pm$ $1.06 \%$ in various drug loaded SPHIPNs. 


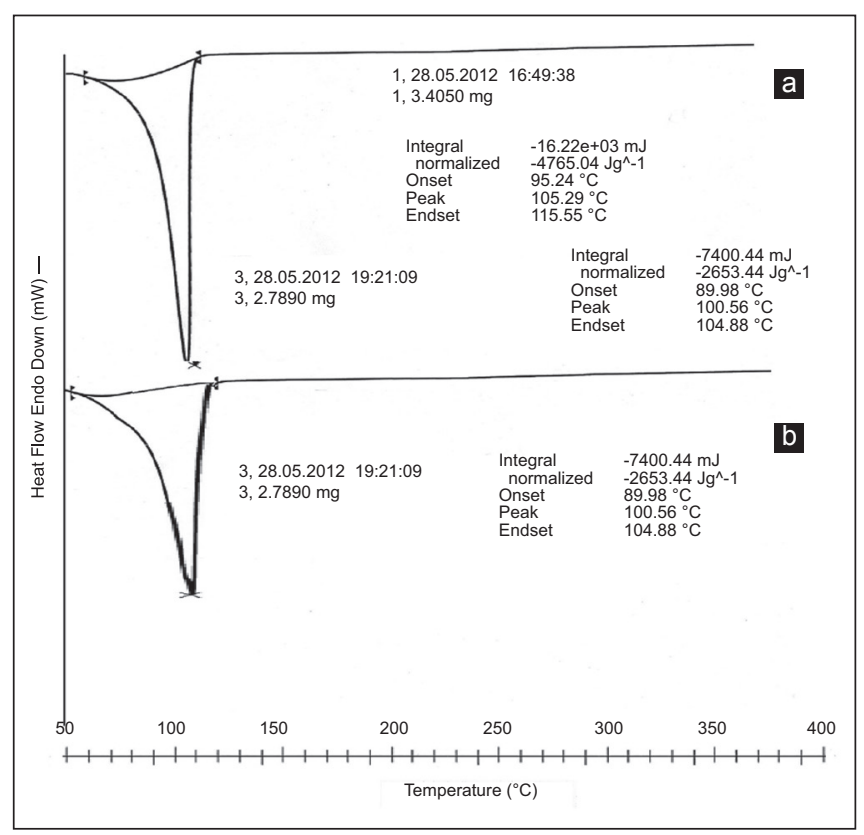

Figure 4: Overlay diagram of differential scanning calorimetry of (a) pure drug (b) drug loaded superporous hydrogels interpenetrating network

\section{DSC}

DSC of pure drug verapamil showed an sharp endothermic peak at $144^{\circ} \mathrm{C}$ with $\Delta \mathrm{H}$ of $12.046 \mathrm{~J} / \mathrm{g}$. The DSC of drug loaded SPHIPNs showed the same endothermic peak, but with decreased intensity [Figure 4], indicating no interaction of drug with hydrogel polymeric network although some polymorphic changes during the formulation may have occurred.

\section{FTIR}

The IR spectra of pure Verapamil exhibits peaks at 2579.67 and $2542.35 \mathrm{~cm}^{1}$ (aldehydic C-H stretching), $2236.67 \mathrm{~cm}^{-1}$ (C-N stretching vibrations), $1592.42 \mathrm{~cm}^{1}, 1518.91 \mathrm{~cm}^{1}$ and $1462 \mathrm{~cm}^{1}$ $\left(\mathrm{C}=\mathrm{C}\right.$ in aromatic ring), $1260.69 \mathrm{~cm}^{1}$ (C-O stretching in aromatic and aliphatic ring), $1026.65 \mathrm{~cm}^{1}$ (C-N aliphatic stretching), $816.97 \mathrm{~cm}^{1}$ and $769.34 \mathrm{~cm}^{1}$ (Meta substituted benzene). The infrared spectra of acrylamide showed the presence of band at $3354.19 \mathrm{~cm}^{1}$ can be assigned to symmetrical and asymmetrical stretching of $\mathrm{N}-\mathrm{H}$ group. The characteristic $\mathrm{C}=\mathrm{O}$ stretching vibration bands of amide and acid groups have been observed at $1673.46 \mathrm{~cm}^{1}$ and $1720 \mathrm{~cm}^{-1}$ respectively and the peak for $\mathrm{CH}=\mathrm{CH}_{2}$ group at $1613.75 \mathrm{~cm}^{-1}$ was observed. The FTIR spectra of HPMC showed the peak at $3421.04 \mathrm{~cm}^{1}$ due to $\mathrm{OH}$ vibrational stretching; symmetric stretching mode of methyl and hydroxyl propyl groups was found at $2928.96 \mathrm{~cm}^{1}$ in which all the $\mathrm{CH}$ bonds extend and contract in phase; the peak at $1621 \mathrm{~cm}^{1}$ indicated the presence of stretching vibration six membered cyclic rings. While, the asymmetric bending vibrations of the methoxy group normally appeared in the region $1500-1450 \mathrm{~cm}^{1}$, the symmetric vibrations were mostly displayed in the range
$1400-1350 \mathrm{~cm}^{1}$. The IR spectrum of sodium alginate exhibited characteristic absorption bands for hydroxyl groups $\left(3430.30 \mathrm{~cm}^{1}\right)$, carboxylate $\left(1416.39 \mathrm{~cm}^{1}\right)$ and carbonyl $\left(1616.03 \mathrm{~cm}^{1}\right)$ [Figure 5]. Cross-linking of alginate by $\mathrm{Ca}^{2+}$ was shown by a decrease in the wavenumber of carbonyl peak from $1616.03 \mathrm{~cm}^{1}$ to $1516.18 \mathrm{~cm}^{1}$. The shift in wavenumber of hydroxyl peak of sodium alginate in hydrogel spectra may be due to a negative effect on bond formation involving adjacent hydroxyl groups as a result of conformational changes of alginate after reacting with $\mathrm{Ca}^{2+}$. The presence of almost all characteristic peaks of drug in hydrogel spectra indicated no interaction of drug with polymeric network.

\section{X-RD}

Diffractogram of Verapamil $\mathrm{HCl}$ showed numerous characteristic peaks at $2 \theta$ of $10.59^{\circ}, 14.45^{\circ}, 17.07^{\circ}, 18.1^{\circ}, 18.84^{\circ}, 20.29^{\circ}, 21.32^{\circ}$, $23.06^{\circ}, 23.75^{\circ}$ and $26.29^{\circ}$. Acrylamide showed the presence of intense crystalline peaks at $12.21^{\circ}$ and $24.31^{\circ}$. X-RD of HPMC showed the amorphous behavior of HPMC due to the presence of broad peaks with the peak of maximum intensity at $5.82^{\circ}$. X-RD of sodium alginate showed amorphous nature of polymer. Figure 6 shows the overlay diagram of X-RD of drug, polymers and drug loaded SPHH. The presence of characteristic peaks of drug in the diffraction pattern of drug loaded SPHIPNs indicated that incorporated drug remains intact in its crystalline state within polymer network. The crystalline nature of the drug was still maintained. However, minor shifts in characteristic peaks and reduced diffraction intensity suggests reduction in quality of the crystals (e.g. change in crystal size) and presence of higher amount of amorphous drug along with polymer.

\section{${ }^{1} \mathrm{H}$ NMR studies}

The presence of various characteristic peaks of pure drug verapamil hydrochloride in proton NMR spectra [Figure 7] of drug loaded SPHIPN indicated drug integrity with in polymer network.

\section{In vitro drug release}

The drug release from six formulation batches (A-F) was carried out to evaluate the effect of i) chemical cross-linker $\left(\mathrm{CaCl}_{2}\right)$ concentration and ii) addition of second polymer (HPMC) on sustain release behavior of drug. The increasing $\mathrm{CaCl}_{2}$ concentration has a pronounced effect on the drug release in first $30 \mathrm{~min}$ (A, C and $\mathrm{E}$ batches) as observed by significant increase in percent cumulative release in these formulations $(22 \%, 44 \%$ and $60 \%$ respectively). The increase in drug release was continued up to $2 \mathrm{~h}$ and then sustained effect was observed at the end of $24 \mathrm{~h}$ [Figure 8]. However increasing $\mathrm{CaCl}_{2}$ has no significant effect on extent of drug release was observed at the end of $24 \mathrm{~h}$ ( $80 \%, 78 \%$ and $74 \%$ respectively). The addition of second polymer (HPMC) lead to significant decrease in drug release at the end of $24 \mathrm{~h}$ (47\%, 23\% and $20 \%$ in B, D and F batches respectively). The significant decrease in drug release by addition of HPMC was observed in batches containing higher concentrations of calcium chloride (batches $\mathrm{D}$ and $\mathrm{F}$ in comparison to $\mathrm{C}$ and $\mathrm{E}$ batches without HPMC). 


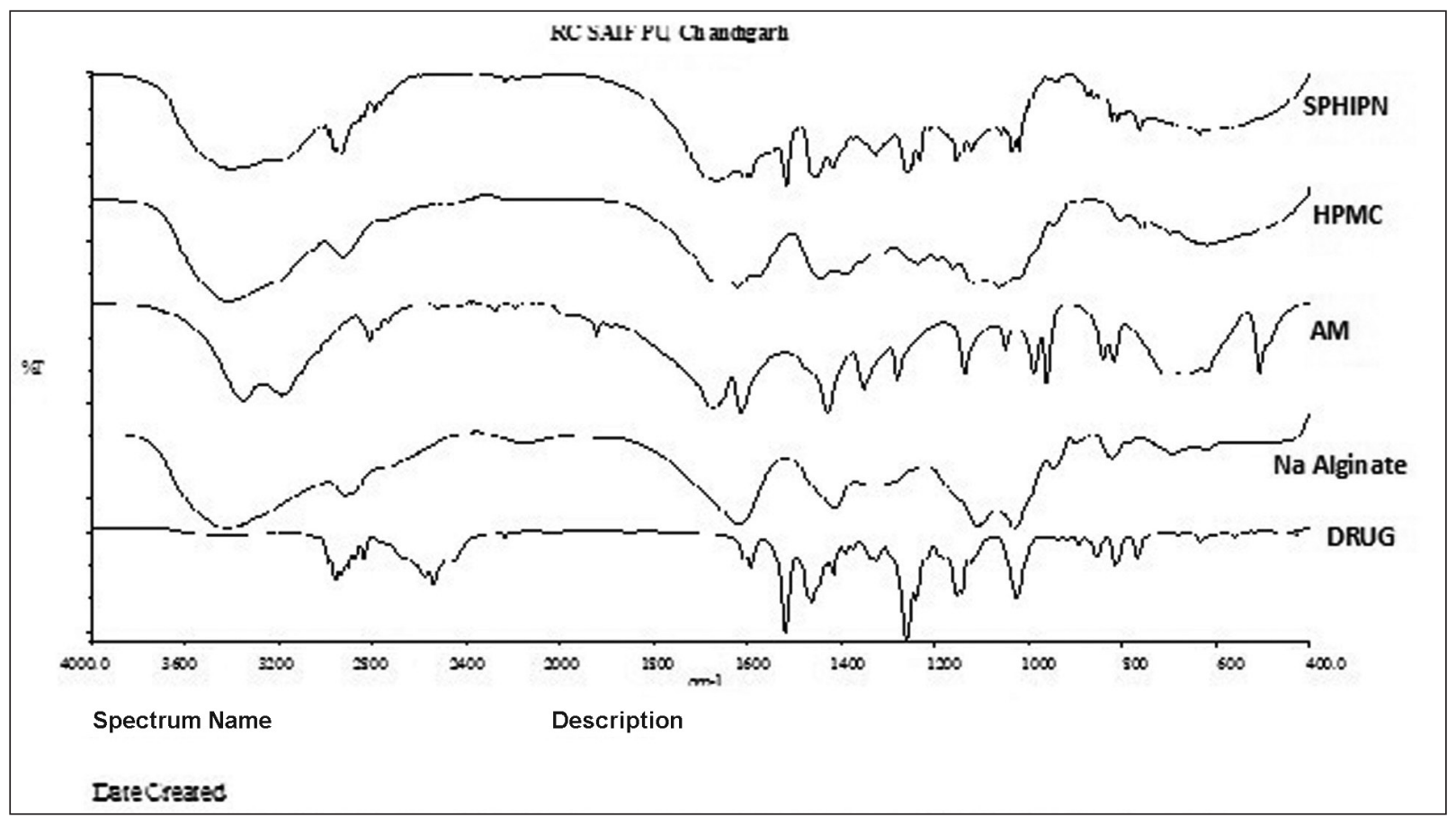

Figure 5: Overlay diagram of Fourier transform infrared spectroscopy of pure drug, sodium alginate, hydroxypropyl methyl cellulose, acrylamide and superporous hydrogels interpenetrating network

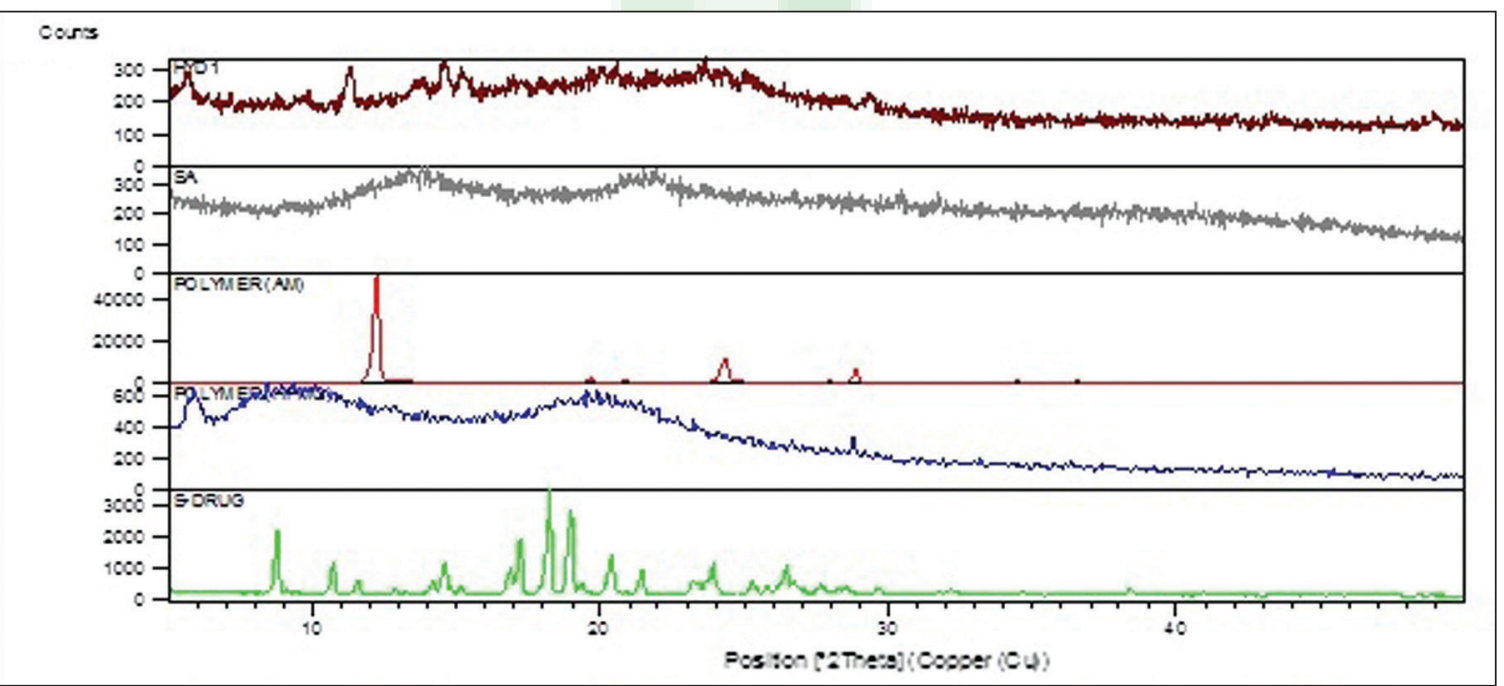

Figure 6: Overlay diagram of X-ray diffraction of pure drug, hydroxypropyl methyl cellulose, acrylamide, sodium alginate and superporous hydrogels interpenetrating network

\section{DISCUSSION}

Various batches of different generation SPHs were synthesized and evaluated for their suitability to act as gastric retention drug delivery systems. Different generations of SPHs were observed for physical characteristics (texture, stickiness, flexibility, hardness etc.) during synthesis. CSPH were observed as sticky during drying, transparent during swelling and highly friable whereas SPHC were comparatively less friable, which may be due to physical cross-linking by second polymer (sodium alginate) into primary polymer (polyacrylamide) network. SPHC when treated with calcium chloride, obtained SPHIPNs were elastic after swelling, which reveals cross-linking of sodium alginate with calcium chloride. This was further confirmed by mechanical strength studies using texture analyzer. Drying conditions during synthesis significantly affected porous network of SPHs. Oven drying completely distorted the porous network during drying as rapid evaporation of water from voids within the network leads to collapse of polymer chains due to high surface tension of water. While, in ethanol 


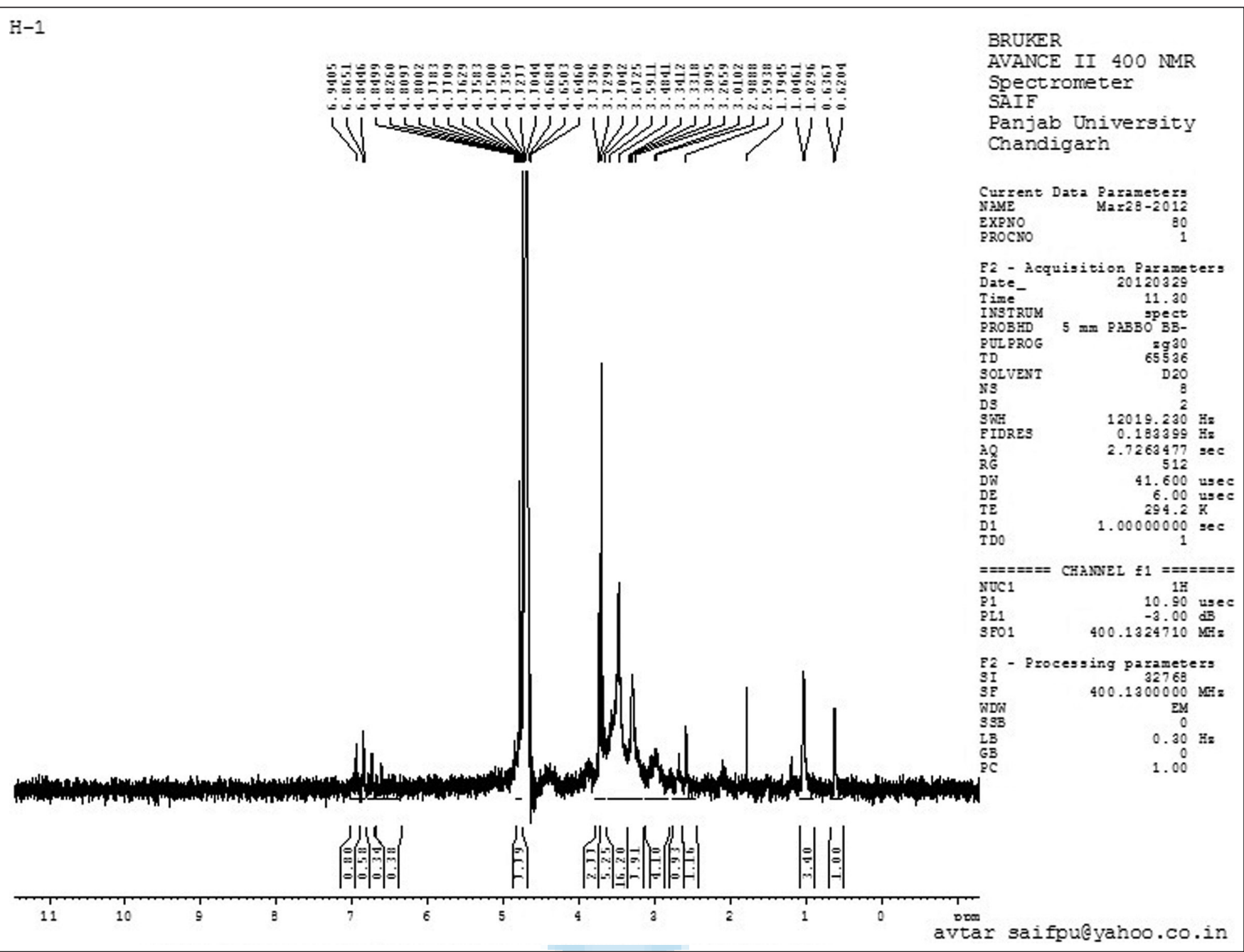

Figure 7: ${ }^{1} \mathrm{H}$ nuclear magnetic resonance spectrum of drug loaded superporous hydrogel interpenetrating network

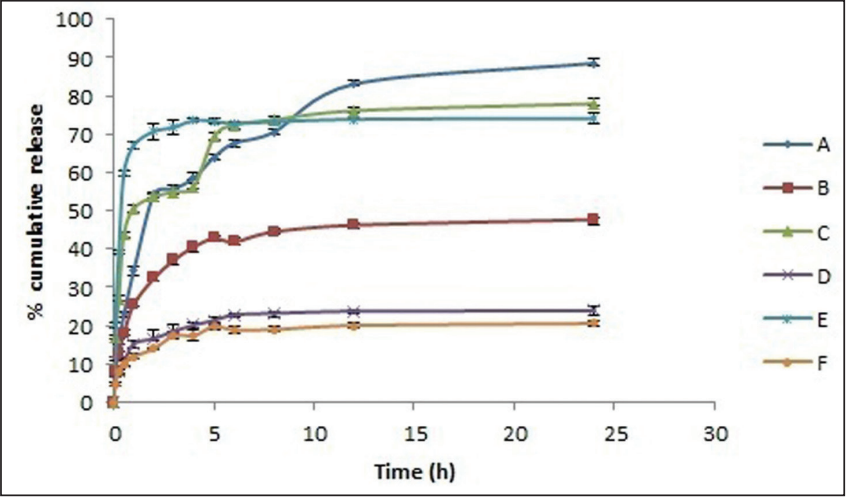

Figure 8: Percent cumulative drug release from various superporous hydrogel interpenetrating network formulations (A-F)

dehydration method ethanol replaces water in void spaces and then is evaporated resulting in intact porous network without shrinkage of capillary channels (due to low surface tension of ethanol). Moreover, during ethanol dehydration, SPH became hard due to precipitation of polymer chains in such poor solvent.
This also contributed to integrity of rigid pore structure (Chen et al., 1999). Freeze drying lead to slight distortion of pores which may be attributed to rapid evaporation of ice crystals. However, prepared SPHs are fluffy (significantly less in weight) yielding better floating characteristics. Equilibrium swelling studies of different generation SPHs revealed maximum equilibrium swelling ratio of $\mathrm{CSPH}$ in comparison to SPHC and SPHIPNs. And equilibrium swelling time was less than $10 \mathrm{~min}$ in CSPH and SPHC whereas it increases to $30 \mathrm{~min}$ by using the different concentrations of chemical cross-linker calcium chloride. The addition of secondary polymer leads to physical cross-linking of polymer network, which strengthens the polymeric network and also maintains the uniformity in interconnected capillary channels as indicated by slight decrease in the equilibrium swelling time and swelling ratio in SPHC. Chemical cross-linking of SPHC by treating with calcium chloride (of different concentration) significantly decreases the equilibrium swelling ratio and time for equilibrium swelling was increased to maximum of $30 \mathrm{~min}$ (in case of SPHIPNs batches treated with $\left.10 \% \mathrm{CaCl}_{2}\right)$. This revealed the crosslinking of alginate portion in polymeric network with $\mathrm{Ca}^{2+}$. The 
equilibrium swelling ratio was decreased by increasing $\mathrm{CaCl}_{2}$ concentration and also leads to surface hardening of SPHIPNs. The increase in mechanical strength by chemical cross-linking was also evaluated using texture analysis studies, which revealed significantly enhanced mechanical strength (more than $10 \mathrm{~N}$ ) in SPHIPNs (treated with $5 \% \mathrm{CaCl}_{2}$ ). Treatment with $\mathrm{CaCl}_{2}$ resulted in the formation of hydrogels with desired mechanical strength by significantly improving the structural integrity of the hydrogel and decreased stress relaxation, which enhanced its ability to withstand pressure of gastric fluids. The increase in calcium chloride concentration (up to 10\%) has no significant impact on mechanical strength rather it affected equilibrium swelling properties significantly (reduced equilibrium swelling ratio and increased equilibrium swelling time). Therefore, for gastric retention purpose, SPHIPN treated with 5\% calcium chloride were observed as optimum batches based on swelling and mechanical properties. The density of various generations of SPHs was observed to be less than 1 , which confirms their floating characteristics. The porosity and void fraction results were also in accordance with swelling studies. The SPHIPNs can act as good reservoir devices for drug candidates as indicated by drug loading data, making them effective gastroretentive drug delivery systems. The drug loaded SPHIPNs were further characterized via DSC, FTIR, $\mathrm{X}$-RD studies to evaluate the interaction of drug with polymer network. The same endothermic peaks of drug in SPHIPN, but of slight decreased intensity confirm drug integrity with in polymeric network. The presence of almost all characteristic peaks of drug in FTIR of SPHIPNs indicates no interaction of drug with other components. X-RD studies further confirm the above results. The in vitro dissolution studies revealed the effect of increased cross-linker concentration on drug release behavior. Significant increase in drug release during $1^{\text {st }} \mathrm{h}$ was obtained with increase in chemical cross-linker $\left(\mathrm{CaCl}_{2}\right.$ concentration), which may be due to more rigid polymeric network at higher concentrations of $\mathrm{CaCl}_{2}$ due to which more free drug will be retained at the surface resulting in initial burst release. However, no effect on extent of drug release at the end of $24 \mathrm{~h}$ was observed by increasing chemical cross-linker concentration as the drug, which gets incorporated in polymeric network will release slowly by diffusion through the polymer gel. HPMC was added as release retardant to obtain sustained release of drug. The drug release was reduced to half (batch $B$ in comparison to batch $\mathrm{A}$ ) or more than half (in batches $\mathrm{D}$ and $\mathrm{F}$ ). On the basis of dissolution data, formulation batch A (treated with $5 \% \mathrm{CaCl}_{2}$ without $\mathrm{HPMC}$ ) was selected as optimum batch with initial fast and then slow release characteristics.

\section{CONCLUSION}

SPH interpenetrating networks (based on acrylamide and sodium alginate) with good swelling and mechanical properties have been evolved to address the need of certain pharmaceutical applications like gastric retention. The ability of these networks to rapidly swell and remain intact in harsh swelling media can be potentially exploited for sustained delivery of drugs. The alginate portion lead to formation of gel networks in the presence of divalent or trivalent metal cations, which is utilized for the formation of SPHIPNs. Different concentrations of calcium chloride were used to get an interpenetrating network with balanced swelling as well as mechanical properties. The prepared SPHIPNs were found to be elastic in swelled state and regain their shape during texture analysis studies. Moreover, the drug release pattern from these SPHIPNs indicated that they can be used where fast onset of action is required (initial burst release) and then the drug is released for next $24 \mathrm{~h}$ or more. The method of drug loading can affect the release behavior of drug from these interpenetrating networks. The results can be further confirmed by in vivo gastric retention analysis by $\mathrm{x}$-ray imaging techniques and in vivo bioavailability studies.

\section{ACKNOWLEDGMENTS}

The authors are grateful to Dr. Madhu Chitkara, Vice Chancellor, Chitkara University, Punjab, India; Dr. Sandeep Arora, Director, Chitkara College of Pharmacy, Chitkara University, Punjab, India; Dr. M.L. Ranga, Vice Chancellor, GJUS\&T, Hisar, India for institutional facilities and technical support. Authors are highly thankful to Prof. Dr. D.N. Yadav, Head, CIPHET, Ludhiana for texture analysis; NIPER, Mohali for DSC studies; Director, CIL, Punjab University for X-RD and FTIR facilities.

\section{REFERENCES}

1. Omidian H, Park K, Rocca JG. Recent developments in superporous hydrogels. J Pharm Pharmacol 2007;59:317-27.

2. Allan $\mathrm{SH}$. Hydrogels for biomedical applications. Adv Drug Deliv Rev 2002;43:3-12.

3. Nagpal M, Singh SK, Mishra DN. Superporous hydrogels as gastroretentive devices. Acta Pharm Sci 2011;53:7-24.

4. Chen J, Park H, Park K. Synthesis of superporous hydrogels: Hydrogels with fast swelling and superabsorbent properties. J Biomed Mater Res 1999;44:53-62.

5. Doorkoosh FA, Brussee J, Verhoef JC, Borchard G, RafieeTehrani M, Junginger HE. Preparation and NMR characterization of superporous hydrogels (SPH) and SPH composites. Polymer 2000;41:8213-20.

6. Chen J, Blevins WE, Park H, Park K. Gastric retention properties of superporous hydrogel composites. J Control Release 2000;64:39-51.

7. Kim SJ, Park SJ, Kim SI. Swelling behavior of interpenetrating polymer network hydrogels composed of poly (vinyl alcohol) and chitosan. React Funct Polym 2003;55:53-9.

8. Rodrigues IR, de Camargo Forte MM, Azambuja DS, Castagno KR. Synthesis and characterization of hybrid polymeric networks (HPN) based on polyvinyl alcohol/chitosan. React Funct Polym 2007;67:708-15.

9. Wang M, Fang $Y$, Hu D. Preparation and properties of chitosanpoly (N-isopropylacrylamide) full-IPN hydrogels. React Funct Polym 2001;48:215-21.

10. Omidian H, Rocca JG, Park K. Elastic, superporous hydrogel hybrids of polyacrylamide and sodium alginate. Macromol Biosci 2006;6:703-10. 
11. Wee S, Gombotz WR. Protein release from alginate matrices. Adv Drug Deliv Rev 1998;31:267-85.

12. Sweetman S. Martindale: The Complete Drug Reference. USA: Pharmaceutical Press, Inc; 2006. p. 1278.

13. Streubel A, Siepmann J, Bodmeier R. Floating matrix tablets based on low density foam powder: Effects of formulation and processing parameters on drug release. Eur J Pharm Sci 2003;18:37-45.

14. Qiu Y, Park K. Superporous IPN hydrogels having enhanced mechanical properties. AAPS PharmSciTech 2003;4:E51.

15. Chavda H, Patel C. Effect of crosslinker concentration on characteristics of superporous hydrogel. Int J Pharm Investig 2011;1:17-21.
16. Vishal Gupta N, Shivakumar HG. Preparation and characterization of superporous hydrogels as gastroretentive drug delivery system for rosiglitazone maleate. Daru 2010;18:200-10.

17. Chavda HV, Patel CN. Preparation and characterization of swellable polymer based superporous hydrogel composites of poly (acrylamide-co-acrylic acid). Trends Biomater Artif Organs 2010;24:83-9.

How to cite this article: Nagpal M, Singh SK, Mishra D. Synthesis characterization and in vitro drug release from acrylamide and sodium alginate based superporous hydrogel devices. Int J Pharma Investig 2013;3:131-40.

Source of Support: Nil. Conflict of Interest: None declared.

\section{“QUICK RESPONSE CODE” LINK FOR FULL TEXT ARTICLES}

The journal issue has a unique new feature for reaching to the journal's website without typing a single letter. Each article on its first page has a "Quick Response Code". Using any mobile or other hand-held device with camera and GPRS/other internet source, one can reach to the full text of that particular article on the journal's website. Start a QR-code reading software (see list of free applications from http://tinyurl.com/yzlh2tc) and point the camera to the QR-code printed in the journal. It will automatically take you to the HTML full text of that article. One can also use a desktop or laptop with web camera for similar functionality. See http://tinyurl.com/2bw7fn3 or http://tinyurl.com/3ysr3me for the free applications. 